\title{
Modeling and Model Predictive Control of Nonlinear Hydraulic System
}

\author{
Petr Chalupa, Jakub Novák \\ Department of Process Control, Faculty of Applied Informatics, Tomas Bata University in \\ Zlin, nám. T. G. Masaryka 5555, 76001 Zlín, CZECH REPUBLIC \\ Email: chalupa@fai.utb.cz
}

\begin{abstract}
This paper deals with modeling and control of a hydraulic three tank system. A process of creating a computer model in MATLAB / Simulink environment is described and optimal PID and model predictive controllers are proposed. Modeling starts with creation of an initial mathematical model based on first principles approach. Further, the initial model is modified to obtain better correspondence with real-time system and parameters of the modified system are identified from measurements. The real time system contains nonlinearities which cannot be neglected and therefore are identified and included in the final mathematical model. Resulting model is used for control design. As the real-time system has long time constants, usage of Simulink model dramatically speeds up design process. Optimal PID and MPC controllers are proposed and compared. Described techniques are not limited to one particular modeling problem but can be used as an illustrative example for modeling of many technological processes.
\end{abstract}

\section{Introduction}

Most of current control algorithms is based on a model of a controlled plant [1]. A plant model can be also used to investigate properties and behavior of the modeled plant without a risk of damage of violating technological constraints of the real plant. Two basic branches of modeling are used in practice: the black box approach and the first principles modeling.

The black box approach to the modeling [2], [3] is based on analysis of input and output signals of the plant and knowledge of physical principle of modeled plant is not required. On the other hand, model obtained by black box approach is generally valid only for signals it was calculated from.

The first principle modeling provides general model which is in optimal case valid for whole range of plant inputs and states. The model is created by analyzing the modeled plant and combining physical laws [4]. On the other hand, there is usually a lot of unknown constants and relations when performing analysis of a plant. Thus, first-principle modeling is suitable especially for simple controlled plants 
with small number of parameters or for obtaining basic information about controlled plant (rage of gain, rank of suitable sample time, etc.).

Combination of both methods is used in the paper. Basic relations between plant inputs and outputs are derived using mathematical physical analysis an obtained model is further improved on the basis of measurements. Obtained relations are used to design a Simulink environment with characteristics as close as possible to the real time system DTS 200 - Three tank System [5]. The major reason for creating the model of this laboratory equipment are big time constants of the plant and thus time consuming experiments. The model can dramatically decrease time needed for controller development because only promising control strategies are applied to the real plant and verified.

The paper is organized as follows. Section 2 presents the modeled system - Amira DTS200. Derivation of initial ideal using first principles modeling and enhancement of this model based on real-time experiments is carried out in Section 3. Section 4 presents the resulting Simulink model in detail and Section 5 copes with PID and MPC control of the plant.

\section{The DTS200 System}

The Amira DTS200 system consists of three interconnected cylindrical tanks, two pumps, six valves, pipes, water reservoir in the bottom, measurement of liquid levels and other elements. The pumps pump water from the bottom reservoir to the top of the left and right tanks. Valve positions are controlled and measured by electrical signals, which allow precise positioning.

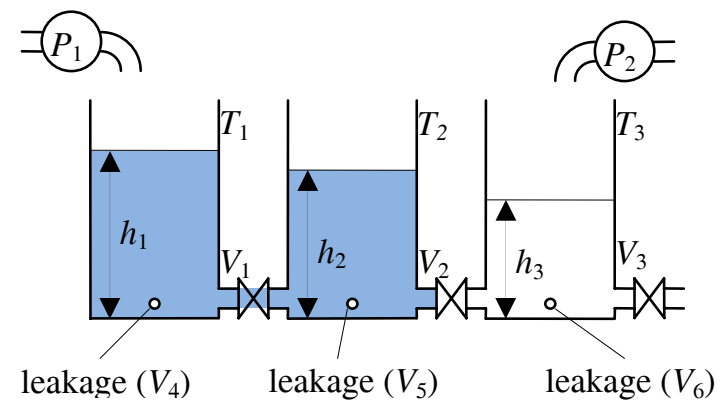

Fig. 1 Scheme of three tank system Amira DTS200

A simplified scheme of the system is shown in Fig. 1. The pump $P_{1}$ controls the inflow to $\operatorname{tank} T_{1}$ while the pump $P_{2}$ controls the liquid inflow to tank $T_{2}$. There is no pump connected to the middle tank $T_{\mathrm{s}}$. The characteristic of the flow between $\operatorname{tank} T_{1}$ and tank $T_{\mathrm{s}}$ can be affected by valve $V_{1}$, flow between tanks $T_{\mathrm{s}}$ and $T_{2}$ can be affected by the valve $V_{2}$ and the outflow of the tank $T_{2}$ can be affected by valve 
$V_{3}$. The system also provides the capability of simulating leakage from individual tanks by opening the valves $V_{4}, V_{5}$ and $V_{6}$.

Pumps are controlled by analogue signals in range from $-10 \mathrm{~V}$ to $10 \mathrm{~V}$. Heights of water level are measured by pressure sensors. Each valve is operated by two digital signals which control motor of particular valve. First signal orders to start closing of the valve while the second signal is used for opening of the valve. If none of the signals is activated the valve remains in its current position. Each valve also provides three output signals: analogue voltage signal correspond to the current position of the valve and two informative logical signals which states that the valve is fully opened or fully closed respectively.

The overall number of inputs to the modeled plant DTS200 is 14:

- 2 analogues signals controlling the pumps,

- 12 digital signals ( 2 for each of the 6 valves) for opening / closing of the valves.

The plant provides 21 measurable outputs which can be used as a control feedback or for measurements of plant characteristics:

- 3 analogue signals representing level heights in the three tanks,

- 6 analogues signals representing position of the valves,

- 12 logical signals ( 2 for each of the 6 valves) stating that corresponding valve is fully opened / closed.

\section{Modeling of the plant}

This section is focused to derivation of mathematical model of the three-tank system end its adaptation to the DTS200 plant using real-time experiments.

\subsection{Ideal model}

This derivation of ideal plant model is based on ideal properties of individual components. The ideal flow of a liquid through a pipe can be derived from Bernoulli and continuity equations for ideal liquid.

Since the flow through a valve depends only on the level difference, the valve position and constants representing pipes and cylindrical tanks, the whole mathematical model can be written as follows: 


$$
\begin{aligned}
& \frac{d h_{1}}{d t}=q_{1}-k_{1} \sqrt{\left|h_{1}-h_{s}\right|} \cdot \operatorname{sign}\left(h_{1}-h_{s}\right)-k_{4} \sqrt{h_{1}} \\
& \frac{d h_{2}}{d t}=k_{1} \sqrt{\left|h_{1}-h_{s}\right|} \cdot \operatorname{sign}\left(h_{1}-h_{s}\right)+k_{2} \sqrt{\left|h_{2}-h_{s}\right|} \cdot \operatorname{sign}\left(h_{2}-h_{s}\right)-k_{5} \sqrt{h_{s}} \\
& \frac{d h_{3}}{d t}=q_{2}-k_{2} \sqrt{\left|h_{2}-h_{s}\right|} \cdot \operatorname{sign}\left(h_{2}-h_{s}\right)-k_{3} \sqrt{h_{2}}-k_{6} \sqrt{h_{2}}
\end{aligned}
$$

where symbols $h_{1}, h_{2}$ and $h_{3}$ represent water level heights in tanks $T_{1}, T_{2}$ and $T_{3}$ respectively, $k$ is a parameter representing valve position and $q$ represents inflow as change of water level in time:

$$
k_{i}=v_{i} \frac{S_{V \max } \sqrt{2 g}}{S_{T}} \quad i=1,2, \ldots, 6 \quad q_{i}=\frac{q_{i}^{\prime}}{S_{T}} \quad i=1,2
$$

The cross-sectional areas of all three tanks are the same and are symbolized by $S_{T}$ This ideal model is successfully used in many control system studies as a demonstration example [6], [7]. Although the ideal model is based on simple equations, analytical solution of the outputs for a given course of inputs is complicated. The problem lies in nonlinearity of equations (1). Also computation of steady state for a given constant inputs is a very complicated task and leads to solution of higher order polynomial equation.

\subsection{Enhanced model}

This section describes enhancement of the initial model and measurement of characteristics of individual parts of DTS200 system.

\section{Characteristics of the pumps}

The characteristics of pumps were measured to refine on (1). The amount of water pumped within certain time was measured for different setting of driving signals $u_{1}$ and $u_{2}$. Characteristics of both pumps are similar and close to linear but do not start at a lower bound ( $u=-1 \mathrm{MU})$ but at least the value of approx. $u=-0.85$ must be applied to the pump to obtain a nonzero output. The maximal pumping of approx. $6 \mathrm{~mm} / \mathrm{s}$ of tank level rise represents $5.37 \mathrm{l} / \mathrm{min}$. Dynamics of the pumps are very fast comparing to other time constants present in the system and therefore were neglected.

\section{Characteristics of the valves}

As stated in Section 2, each of plant's 6 valves is driven by two dedicated logical signals. These signals are used for starting valve's motor in closing or opening direction respectively. If none signal is activated the valve remains in its current po- 
sition. Each valve provides three output signals. The current valve position is determined by analogue signal. Higher values of signal represent closed valve and lower values represent opened valve. The other two signals are logical and state that valve is opened or closed respectively. Valve characteristics are studied in more detail in [9].

\section{Valve flow parameters for valves}

Valve flow parameters $k_{i}$ as appeared in (1) were computed from measurements of draining through individual valves which are connected to outflow pipes $\left(V_{3}, V_{4}\right.$, $V_{5}$ and $V_{6}$ ). The draining of a tank to the reservoir situated below the tanks is described by differential equation

$$
\frac{d h(t)}{d t}=-k \sqrt{h(t)+h_{0}} \quad h(t)=\frac{k^{2}}{4} \cdot t^{2}-k \sqrt{h(0)+h_{0}} \cdot t+h(0)
$$

where $h(0)$ is initial water level and $h_{0}$ is the vertical length of outflow pipe. Due to mechanical configuration of the plant, the value of $h_{0}$ for outflow valves $V_{3}, V_{4}$, $V_{5}$, and $V_{6}$ cannot be measured directly. But it can be identified from draining course. The valve was partially closed to different positions at the beginning of draining experiment and relation between valve position and value of $k$ was achieved.

Similar approach to obtaining values of $k$ can be used also for valves $V_{1}$ and $V_{2}$ which interconnect tanks $T_{1}$ and $T_{2}$, and $T_{2}$ and $T_{3}$, respectively.

Valve hysteresis

Experiments unveiled a hysteresis present in all valves. The value of valve position itself does not give sufficient information about current value of parameter $k_{2}$. As can be seen from Fig. 2, if the position is $0 \mathrm{MU}$ the value of $k_{2}$ can be anywhere in range 0.03 to 0.13 . Especially in case of using the valve as an actuator the hysteresis should be taken into account. Otherwise control process can easily become unstable.

\section{Modeling of valve characteristics}

The course of relation between valve position in MU and $k$ is similar to step responses of dynamical system and therefore it was modeled in similar way. A model based on transfer of $4^{\text {th }}$ order aperiodic system produced satisfactory results. Thus relation between position and $k$ border curve was as follows:

$$
\begin{array}{cc}
\operatorname{pos}<\operatorname{pos}_{0}: \quad k=k_{\max }\left[1-\frac{1}{6} e^{\frac{-b}{a}} \frac{b^{3}+3 b^{2} a+6 b a^{2}+6 a^{3}}{a^{3}}\right] \\
\quad b=p o s_{0}-p o s \\
\operatorname{pos} \geq \operatorname{pos}_{0}: \quad k=0
\end{array}
$$


where pos is valve position in MU and parameters $a$ and $\operatorname{pos}_{0}$ were obtained by nonlinear regression. The regression for valve $V_{2}$ is presented in Fig. 2 .

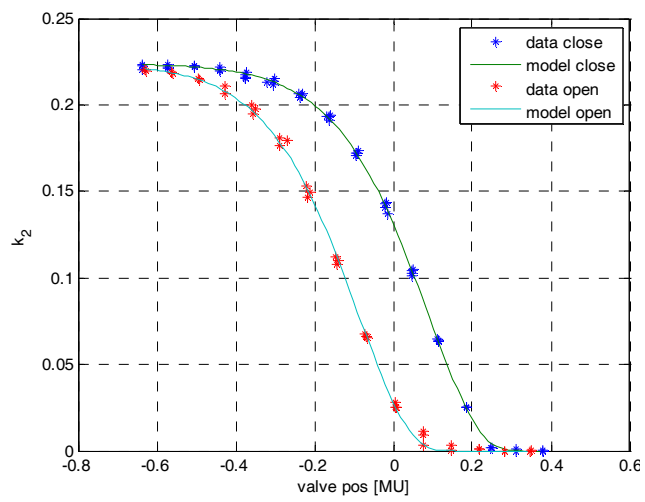

Fig. 2 Model of parameter $k$ for valve $V_{2}$

\section{Simulink model}

All the models of individual parts of the DTS200 plant were incorporated into single block in MATLAB / Simulink environment. The block has the same inputs and outputs as the real plant. Thus it contains all 14 inputs and 21 outputs described in Section 2. The Simulink block of resulting model of DTS200 plant is depicted in Fig. 3.

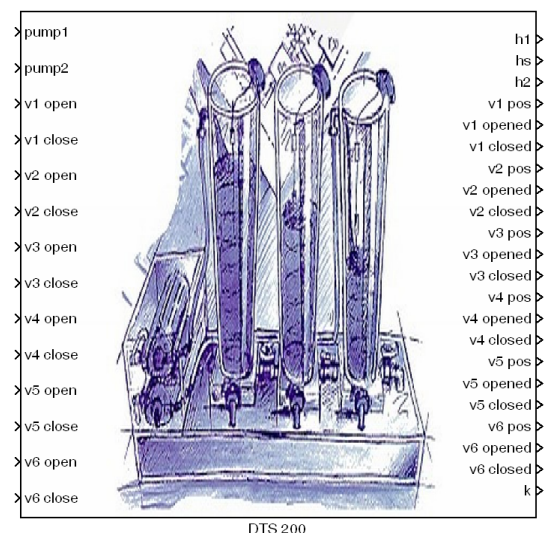

Fig. 3 Block of Simulink model of DTS200

The model is designed as masked subsystem where only necessary initial states can be entered by user. The initial states are: initial water level in individual tanks and initial valve positions and corresponding values of valve parameters $k$. 
Masked subsystems and subsystems are used also to model individual parts of the plant. For example, internal structure of valve state subsystem is presented in Fig. 4. This hierarchical structure is useful to maintain lucidity.

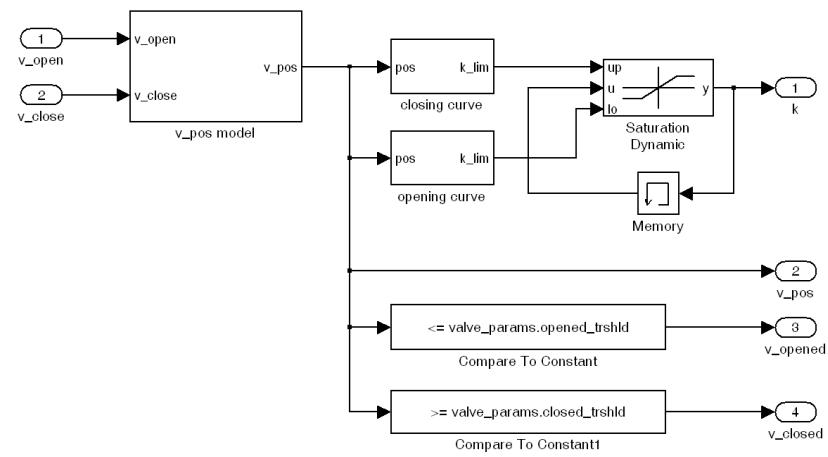

Fig. 4 Internal structure of valve state subsystem

Detailed description of the Simulink model can be found in [10].

\section{PID and model predictive control}

The control experiment was carried out to verify the Simulink model and to find out how the controllers cope with nonlinearities in the plant.

The experiment configuration was following: there was constant inflow to tank $T_{1}$ by pump $P_{1}$. The valve $V_{1}$ between tanks $T_{1}$ and $T_{2}$ was opened to a constant position. Valves $V_{4}$ (leakage from tank $T_{1}$ ) and $V_{2}$ (interconnection of tanks $T_{2}$ and $T_{3}$ ) were closed. The goal of the control was to drive water level in tank $T_{2}$ using valve $V_{5}$ as an actuator. It should be noted that characteristics of valve $V_{5}$ is nonlinear and contains big hysteresis.

The plant was in steady state at the beginning of all experiments. The valve V5 was partly opened and initial water levels were as follows:

$$
\begin{aligned}
& h_{1}(0)=235 \mathrm{~mm} \\
& y(0)=h_{2}(0)=151 \mathrm{~mm} \\
& u(0)=30 \%
\end{aligned}
$$

The valve position was controlled by a simple internal controller connected to valve drive. This internal control loop is driven by required valve position signal. This control signal is in percentage where $0 \%$ corresponds to fully closed valve (i.e. step change of $v 5$ closed signal) and $100 \%$ represents fully opened valve (i.e. 
step change of $v 5$ opened signal). Sample time of $T_{s}=0.1 \mathrm{~s}$ was used for measurements but all the controller used sample period of $T_{\mathrm{c}}=5 \mathrm{~s}$.

Quadratic criterion was used to compare individual courses from the control error point of view:

$$
J_{e}=\sum_{i=1}^{N} e^{2}(i)=\sum_{i=1}^{N}[w(i)-y(i)]^{2}
$$

Usually control signals differences are penalized of evaluated. But in case of DTS200 crucial problem is in starting and stopping the valve motor because often starting of the valve decreases its durability. The displacement of the valve is not as important as the fact that the valve motor had to be started. Valve starts were measured using following criterion:

$$
J_{u}=\sum_{i=1}^{N-1}\left[v 5 \_ \text {open }(i+1)-v 5 \_ \text {open }(i)>0\right]+\sum_{i=1}^{N-1}\left[v 5 \_ \text {close }(i+1)-v 5 \_ \text {close }(i)>0\right] \text { (7) }
$$

\section{PID control}

First of all the plant was controlled by classical PID controller. Controller parameters were tuned to minimize criterion (6). A MATLAB function fminsearch was used for this task. This function uses Nelder-Mead Simplex Method to find criterion minimum [11]. Resulting courses are depicted in Fig. 5. It can be seen that even the valve position (control signal) changes smoothly, the output is not so smooth. This corresponds to overcoming of valve's hysteresis.

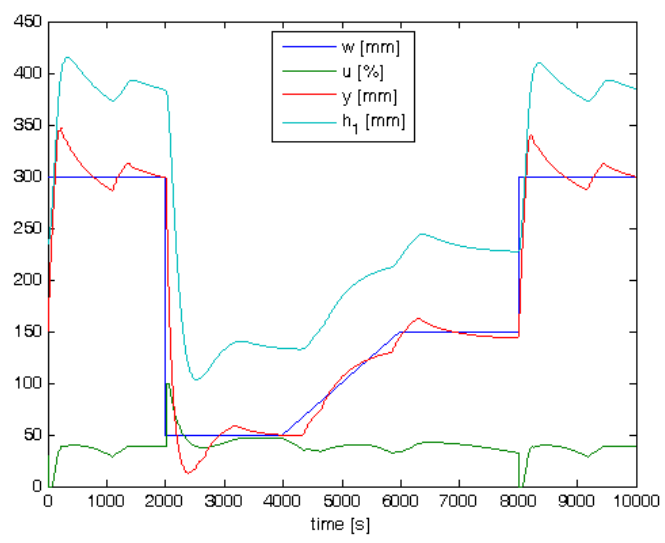

Fig. 5 Optimal PID control DTS200

\section{Model predictive control}

A linear model of the system was identified by applying random signal to its input and parameters of the linearized model were used in MPC controller. The Simulink control scheme is presented in Fig. 6. 


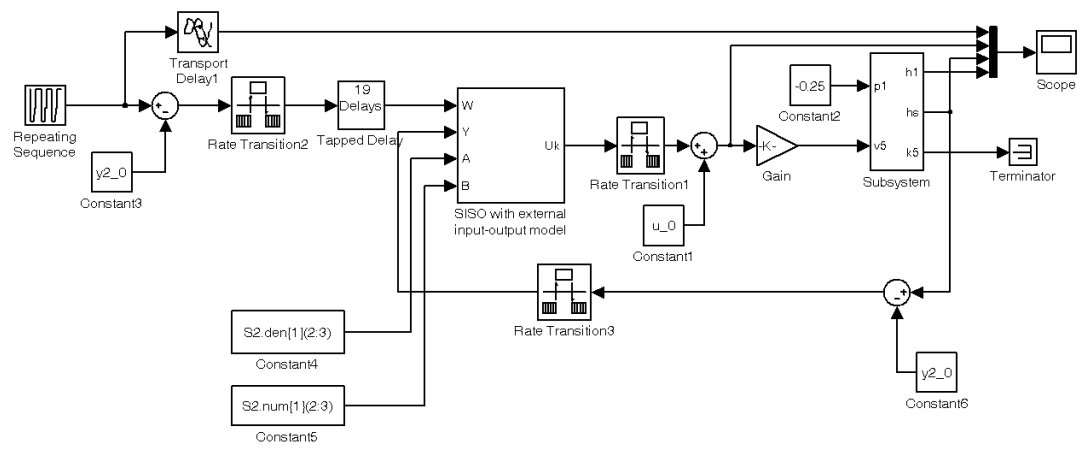

Fig. 6 MPC scheme

A controller from the STuMPCoL library [12] was used to perform the control task. A quadratic criterion with both control and prediction horizon equal to 20 samples was used to compute control signals and receding horizon strategy was used [13]. The resulting control courses are presented in Fig. 7.

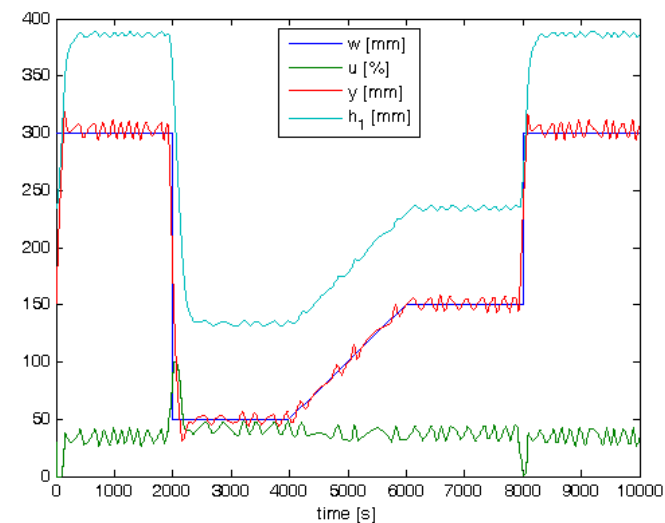

Fig. 7 Control courses using MPC

It was observed that the MPC copes better with crossing of the hysteresis but on the other hand control signal was oscillating around steady states .

Values of criteria defined by equations (6) and (7) are summed up in the Table 1. The MPC was more accurate but it also has slightly actuator demands.

Table 1. Control criterions

\begin{tabular}{lll}
\hline controller & $J_{\mathrm{e}}$ & $J_{u}$ \\
\hline PID & 778 & 3118 \\
\hline MPC & 290 & 5396 \\
\hline
\end{tabular}




\section{Conclusion}

The paper presents Simulink model of hydraulic system. The Amira DTS200 three tank system was considered but used techniques can be easily generalized to a wide set of hydraulic systems. Despite simplicity of the ideal model, real-time system contains several nonlinearities which incorporate complexity to the system. Especially hysteresis of the valves plays a big role in the plant behavior.

A PID and MPC controllers were designed to control the system and verified usability of the model for the controller design. Further improvement of control courses can be reached by usage of nonlinear controller.

Acknowledgments This work was supported by the Ministry of Education of the Czech Republic under grant 1M0567 and by the European Regional Development Fund under the project CEBIA-Tech No. CZ.1.05/2.1.00/03.0089.

\section{References}

1. V. Bobál, J. Böhm, J. Fessl \& J. Macháček:. Digital Self-tuning Controllers: Algorithms, Implementation and Applications (Springer - Verlag London Ltd., 2005).

2. G. P. Liu, Nonlinear identification and control - A neural network Approach (Springer Verlag London Ltd., London, 2001).

3. L. Ljung, System identification : theory for the user (Upper Saddle River, NJ : Prentice Hall PTR, 1999)

4. D. M. Himmelblau, J. B. Riggs, Basic principles and calculations in chemical engineering (Upper Saddle River, N.J. : Prentice Hall, 2004)

5. Amira, DTS200 Laboratory Setup Three - Tank - System (Amira GmbH, Duisburg, 2002).

6. L.Li \& D. Zhou, Fast and robust fault diagnosis for a class of nonlinear systems: detectability analysis, Computers \& Chemical Engineering, 28, 2004, 2635-2646.

7. D. Henry \& A. Zolghadri, Norm-based design of robust FDI schemes for uncertain system under feedback control: Comparison of two approaches, Control engineering practice, 14, 2006, 1081-1097.

8. Humusoft, Real Time Toolbox (Humusoft, Praha, 2011), online: http://www.humusoft.cz/produkty/rtt/.

9. P. Chalupa, J. Novák, V. Bobál, Modeling of Hydraulic Control Valves. In. Proceedings of the 13th WSEAS International Conference (ACMOS '11), Lanzarote, Canary Islands, Spain, May 27-29, 2011. pp. 195-200.

10. P. Chalupa, J. Novák, V. Bobál. Comprehensive Model of DTS200 Three Tank System in Simulink. International Journal of Mathematical Models and Methods in Applied Sciences, 6 (2), 2012, pp.358-365.

11. J.C. Lagarias, J. A. Reeds, M. H. Wright, and P. E. Wright, "Convergence Properties of the Nelder-Mead Simplex Method in Low Dimensions," SIAM Journal of Optimization, 9(1), 1998, pp. 112-147.

12. Chalupa, P. STuMPCoL Self-Tuning Model Predictive Controllers Library [on line]. http://www.fai.utb.cz/people/chalupa/STuMPCoL, 2012

13. W.H. Kwon, S. Han,. Receding Horizon Control. London: Springer-Verlag, 2005. 380 pages. 978-1-84628-024-5. 